
2 Research Square
Preprints are preliminary reports that have not undergone peer review.
They should not be considered conclusive, used to inform clinical practice, or referenced by the media as validated information.

\title{
Effect of preoperative biliary drainage in patients with moderately severe obstructive jaundice on the outcomes of pancreatoduodenectomy: A meta- analysis study
}

\author{
Xiaolei Wang \\ Jilin University \\ Shanshan Meng \\ Jilin University First Hospital \\ Yaowei Hu \\ Jilin University First Hospital \\ Kehang Duan \\ Jilin University First Hospital \\ Feng Wei (D630824099@qq.com)
}

Research article

Keywords: preoperative biliary drainage; obstructive jaundice; pancreatoduodenectomy; meta-analysis

Posted Date: December 12th, 2019

DOl: https://doi.org/10.21203/rs.2.18871/v1

License: () (1) This work is licensed under a Creative Commons Attribution 4.0 International License. Read Full License 


\section{Abstract}

Background The purpose of this meta-analysis was to examine the impact of preoperative biliary drainage (PBD) on the perioperative outcomes of pancreatoduodenectomy (PD) in patients with total bilirubin $>100$ umol/L.Methods In this meta-analysis, studies that compared the perioperative outcomes of PBD and non-PBD patients with total bilirubin $>100$ umol/L, and were published in EMBASE, PubMed, the Cochrane library, Web of Science, VIP database, Wanfang data, Chinese biomedical literature and CNKI database from inception up to October 2019 were included. The odds ratios (OR) or mean differences were calculated with 95\% confidence intervals (Cl).Results Nine trials with 744 patients, which compared PBD (267 patients) with non-PBD (477 patients), were included. There was no significant difference in perioperative mortality between these two groups (OR: $0.51,95 \% \mathrm{Cl}: 0.19$ to $1.39 ; \mathrm{P}=0.19)$. Postoperative hospital stay (mean difference: $-2.35,95 \% \mathrm{Cl}:-3.70$ to $-1.00 ; \mathrm{P}=0.0007$ ), operating time (mean difference: $-33.03,95 \% \mathrm{Cl}:-44.14$ to 21.93 ; $\mathrm{P}<0.00001$ ), estimated blood loss (mean difference: $-141.18,95 \% \mathrm{Cl}:-213.25$ to $-69.11 ; \mathrm{P}=0.0001)$ and overall morbidity (OR: $0.68, \mathrm{Cl}: 0.48$ to $0.95 ; \mathrm{P}=0.02)$ were significantly lower in the PBD group than in the non-PBD group.Conclusion Patients who received PBD had similar perioperative mortality, but had decreased postoperative hospital stay, operating time, estimated blood loss and overall morbidity, when compared to patients without PBD. Therefore, PBD should be routinely performed for patients planned for PD with a total bilirubin of $>100 \mathrm{umol} / \mathrm{L}$.

\section{Background}

Malignant obstructive jaundice occurs due to luminal obstruction of the extrahepatic bile duct by tumors, such as cholangiocarcinoma, pancreatic cancer and periampullary carcinoma. Surgical excision remains as the only curative treatment for such cases. Studies have shown that obstructive jaundice can lead to liver metabolic dysfunction ${ }^{1}$, renal failure ${ }^{2}$ and decreased immune functions ${ }^{3}$, thereby resulting in higher postoperative complications. Shaib ${ }^{4}$ et al. studied 2,306 patients with obstructive jaundice, and found that preoperative biliary drainage (PBD) was helpful in reducing postoperative morbidities. However, McPhern ${ }^{5}$ et al. reported that the mortality of obstructive jaundice with PBD was distinctly higher than that of non-PBD patients. To date, the indications and criteria for PBD in obstructive jaundice patients who require surgery remains unclear.

The present study aims to explore the impact of PBD in patients with moderately severe obstructive jaundice (total bilirubin $>100$ umol/L) on perioperative outcomes of pancreatoduodenectomy (PD).

\section{Methods}

\section{Search strategy}

Relevant studies were retrieved from the databases, including EMBASE, PubMed, the Cochrane library, Web of Science, VIP database, Wanfang Data, Chinese biomedical literature and CNKI database, from inception up to October 2019, using the following search terms: "preoperative biliary drainage", "obstructive jaundice" and "pancreaticoduodenectomy". Then, a systematic review was conducted following the Preferred Reporting Items for Systematic Reviews and Meta Analyses (PRISMA) ${ }^{6}$ and Cochrane Collaboration ${ }^{7}$ guidelines.

\section{Selection criteria}

Studies that satisfied the following criteria were included in the present meta-analysis: original articles reported in the English or Chinese language; studies on patients with obstructive jaundice (total bilirubin > 100 umol/L), who underwent pancreaticoduodenectomy; studies with PBD and non-PBD groups; studies that reported the perioperative mortality, hospital stay, operating time, estimated blood loss and overall morbidity. The exclusion criteria were as follows: studies with irretrievable data; non-comparative studies; letter to editors, comments, or meta-analyses; studies with duplication of data; studies with < 10 patients in either group.

\section{Data extraction}

Two investigators independently extracted the data. All discrepancies were solved by consensus, or with the help of a third investigator. The following information were extracted from each study: study groups, author, year of publication, total bilirubin, number of patients, perioperative mortality, hospital stay, operating time, estimated blood loss and overall morbidity.

\section{Statistical analysis}

The present meta-analysis was conducted according to the guidelines of the Cochrane Handbook for Systematic Reviews of Interventions 5.1.0. The observed indexes were expressed as an odds ratio (OR) with 95\% confidence interval (Cl) for dichotomous variables, and the mean difference was expressed with $95 \% \mathrm{Cl}$ for continuous variables. The Mantel-Haenszel method was applied to combine the OR, and the inverse variance was used to merge the mean difference for the observed indexes. The heterogeneity among studies was evaluated using a standard $X^{2}$-test with the standard of significance set at a $P$ value of $<0.05$, and assessed via the $R$ statistic with the standard of significance set at $R>30 \%$. If heterogeneity was present, a random effects model was applied for the meta-analysis. If heterogeneity was absent, a fixed effects model was used. The OR or mean difference with $95 \% \mathrm{Cl}$, and the heterogeneity of either of the subgroups were respectively calculated. The subgroup differences were assessed, and $P<0.05$ was considered representative of statistical 
significance. All statistical analyses were carried out using Review Manager Version 5.3. For assessing the quality of these studies, bias exploration using a funnel plot and Egger's regression method was planned, but this could not be performed due to the small number of trials included in the present review.

\section{Results}

After the initial search in all these databases, a total of 881 studies were retrieved. After the preliminary screening and elimination of articles with duplication of data, 89 articles were selected. After reading the full text of these articles and excluding articles from which data could not be extracted, nine articles (Table 1, Fig. 1) were selected $^{8-16}$.

\begin{tabular}{|c|c|c|c|c|c|c|}
\hline \multirow[t]{2}{*}{ Reference } & \multirow[t]{2}{*}{ Year } & \multirow[t]{2}{*}{ Country } & \multicolumn{2}{|c|}{ Cases } & \multirow[t]{2}{*}{ Outcome indicators } & \multirow[t]{2}{*}{ Total bilirubin (umol/L) } \\
\hline & & & PBD & Non-PBD & & \\
\hline Chen D. & 2002 & China & 34 & 64 & प्याए। & $110-385$ \\
\hline Wang J.X. & 2011 & China & 33 & 30 & प्राप्रा & $140-410$ \\
\hline Bi X.Y. & 2004 & China & 22 & 30 & प्राप्रा & $>170$ \\
\hline Sang W.C. & 2009 & China & 32 & 59 & प्या० & $140-410$ \\
\hline Wen Y.Z. & 2015 & China & 35 & 30 & प्राप्रा & $120-380$ \\
\hline Wang X.Z. & 2018 & China & 27 & 107 & प्राप्रा & $>170$ \\
\hline Ma E.G. & 2013 & China & 21 & 61 & प्र & $>170$ \\
\hline Zhao P. & 2016 & China & 40 & 38 & 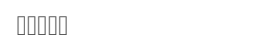 & $>170$ \\
\hline Sewnath, M.E & 2001 & Netherlands & 23 & 58 & प्राप्र & $>100$ \\
\hline
\end{tabular}

Table 1 Clinical characteristics of the included nine articles: $₫$ mortality; $₫$ postoperative hospital stay; $₫$ operating time; $\bigotimes$ estimated blood loss; $₫$ overall morbidity

Figure 1 The flowchart of the selection process for identifying relevant articles for the meta-analysis

\section{Mortality}

Ten articles with 679 patients contained data on perioperative mortality. Since the heterogeneity among these studies was absent $(P=0 \%, P=0.99)$, the fixed effects model was used. The perioperative mortality was $1.72 \%$ in PBD patients and $4.03 \%$ in non-PBD patients, but the difference was not statistically significant (OR: $0.51,95 \% \mathrm{Cl}: 0.19$ to $1.39 ; P=0.19 ; \mathrm{Fig}$. 2 ). In the subgroup analysis, the prospective studies (OR: $0.95, \mathrm{Cl}: 0.06$ to $15.73 ; P=0.97)$ and retrospective studies (OR: $0.47, \mathrm{Cl}: 0.16$ to $1.39 ; P=0.17)$ yielded similar results (Fig. 3$)$. There was no significant heterogeneity for all researches $(R=0 \%$, $P=0.99)$. The heterogeneity was insignificant for retrospective researches $(P=0 \%, P=0.99)$. There was no subgroup distinction among the prospective and retrospective studies $(P=0.65)$.

Figure $2 \mathrm{~A}$ forest plot of the individual data and pooled estimate of perioperative mortality in studies that have PBD and non-PBD groups. The MantelHaenszel fixed effect model was applied, and the ORs were presented with $95 \% \mathrm{Cl}$.

Figure 3 A forest plot of the pooled estimate of perioperative mortality in prospective and retrospective studies. The Mantel-Haenszel method was applied.

\section{Postoperative hospital stay}

There was significant difference (mean difference: $-2.35,95 \% \mathrm{Cl}:-3.70$ to $-1.00 ; P=0.0007 ; \mathrm{Fig} .4$ ) in postoperative hospital stay between the two groups. There was no change in the results when a random effects model was used. The heterogeneity was not statistically significant $(P=0 \%, P=0.58)$. Both prospective studies (mean difference: $-4.07, \mathrm{Cl}:-7.32$ to $-0.81 ; P=0.01$ ) and retrospective studies (mean difference: $-1.98, \mathrm{Cl}:-3.54$ to $-0.42 ; P=0.01$ ) produced similar outcomes (Fig. 5). There was no pregnant heterogeneity among all the studies $(R=44 \%, P=0.11)$. The heterogeneity was meaningless for prospective studies $(P=60 \%, P=0.11)$ and retrospective studies $(R=0 \%, P=0.69)$. There was no subgroup distinction between prospective studies and retrospective studies $(P=0.26)$. 
Figure $4 \mathrm{~A}$ forest plot of the individual data and pooled estimate of postoperative hospital stay in the included studies. The inverse variance fixed effect model was applied. The mean difference was presented with $95 \% \mathrm{Cl}$.

Figure $5 \mathrm{~A}$ forest plot of the pooled estimate of postoperative hospital stay in prospective and retrospective studies. The inverse variance method was applied.

\section{Operating time}

Six studies with 529 patients revealed that non-PBD patients had a distinctly longer operating time, when compared to PBD patients. However, there was no distinct heterogeneity between all six studies $(R=0 \%, P=0.68)$. In the fixed effect model, the distinction was statistically significant, and the combined mean difference was -33.03 (95\% Cl: -44.14 to $21.93 ; P<0.00001$; Fig. 6 ). In the subgroup analysis, according to the study design, the prospective studies (mean difference: $-38.12,95 \% \mathrm{Cl}:-54.34$ to $-21.90 ; P<0.00001)$ and retrospective studies (mean difference: $-28.55,95 \% \mathrm{Cl}:-43.78$ to $-13.31 ; P=0.0002)$ produced similar results (Fig. 7). The heterogeneity was meaningless for both prospective studies $\left(P^{2}=16 \%, P=0.27\right)$ and retrospective studies $(P=0 \%, P=0.74)$. There was no subgroup distinction between prospective studies and retrospective studies $(P=0.40)$.

Figure $6 \mathrm{~A}$ forest plot of the individual data and pooled estimate of operative time in the included studies. The inverse variance fixed-effect model was applied. The mean difference was presented with $95 \% \mathrm{Cl}$.

Figure $7 \mathrm{~A}$ forest plot of the pooled estimate of operative time in the prospective and retrospective studies. The inverse variance method was applied.

\section{Estimated blood loss}

Six studies reported data on estimated blood loss in PBD vs. non-PBD patients. The heterogeneity among studies was significant $(R=83 \%, P<0.00001)$. In the random effects model, the estimated blood loss was significantly lower in the PBD group (mean difference: $-141.18,95 \%$ Cl: -213.25 to $-69.11 ; P=0.0001$; Fig. 8). In the pooled analysis of retrospective studies, intraoperative blood loss was significantly lower in PBD patients (mean difference: $-205.78,95 \%$ Cl: -239.16 to $-172.40 ; P<0.00001$ ), while prospective studies (mean difference: $-107.0095 \% \mathrm{Cl}:-246.11$ to $32.10 ; P=0.13$ ) did not reveal any significant difference in estimated blood loss between the two groups (Fig. 9). The heterogeneity was significant for prospective studies $(R=94 \%, P<0.00001)$, but was not significant for retrospective studies $(R=0 \%, P=0.42)$. There was no subgroup distinction between prospective and retrospective studies $(P=0.18)$.

Figure $8 \mathrm{~A}$ forest plot of the individual data and pooled values of estimated blood loss in studies with PBD and non-PBD groups. The inverse variance fixed effects model was applied. The mean difference was presented with $95 \% \mathrm{Cl}$.

Figure $9 \mathrm{~A}$ forest plot of the pooled values of estimated blood loss in prospective and retrospective studies. The inverse variance method was applied.

\section{Overall morbidity}

The morbidity rate was $35.96 \%$ (96 of 267 ) for PBD patients and $44.44 \%$ (212 of 477 ) for non-PBD patients, and the difference was statistically significant (OR: $0.68, \mathrm{Cl}: 0.48$ to $0.95 ; P=0.02 ; \mathrm{Fig} .10)$. There was no change in outcomes when a random effects model was applied. The heterogeneity was not significant $(R=0 \%, P=0.57)$. Retrospective studies had a trend towards decreased overall morbidity in PBD patients (OR: $0.52, \mathrm{Cl}: 0.30$ to $0.93 ; P=0.03)$, while prospective studies (OR: $0.66, \mathrm{Cl}: 0.32$ to $1.39 ; P=0.28$ ) had no significant difference between the two groups (Fig. 11). There was no distinct heterogeneity for whole researches $(R=35 \%, P=0.14)$. The heterogeneity was not significant for both prospective studies $(R=0 \%, P=0.50)$ and retrospective studies $(P=48 \%, P=0.07)$. There was no subgroup distinction among prospective studies and retrospective studies $(P=0.62)$.

Figure $10 \mathrm{~A}$ forest plot of the individual study proportions and pooled estimate of overall morbidities in the PBD and non-PBD groups. A Mantel-Haenszel fixed effects model was applied. The odds ratios were presented with $95 \% \mathrm{Cl}$. 
Figure $11 \mathrm{~A}$ forest plot of the pooled values of overall morbidities in prospective and retrospective studies. The Mantel-Haenszel method was applied.

\section{Sensitivity analysis}

Since these prospective and retrospective studies were analyzed for mortality, postoperative hospital stay and overall morbidity, a sensitivity analysis to subtotal the plots by prospective studies $v s$. retrospective studies was performed. There were no significant differences in OR or mean difference between these prospective and retrospective studies (all, P>0.05; Figs. 3, 5, 7, 9 and 11).

\section{Discussion}

Studies conducted in the end of the $20^{\text {th }}$ century revealed that upfront surgery in patients with obstructive jaundice is associated with increased postoperative complications and mortality. ${ }^{18,19}$ Hence, PBD became a routine procedure for such patients. However, subsequent studies in the $21^{\text {st }}$ century revealed that PBD itself leads to various complications, especially infectious complications ${ }^{20}$. The positive rate of bile culture after an operation was also found to increase ${ }^{21}$. In order to resolve this controversy, this meta-analysis was conducted to determine the impact of PBD in patients with total bilirubin $>100$ umol/L, in terms of postoperative mortality, hospital stay, operating time, estimated blood loss and overall morbidity.

The main causes of postoperative death in patients with obstructive jaundice are infection and hemorrhage. Studies have shown that high bilirubin levels decrease the body" immunity and increase the risk of infection ${ }^{22,23}$. PBD can effectively reduce the serum bilirubin levels, and help restore appetite, reduce symptoms and improve the immune status. However, in the present study, no statistical difference in perioperative mortality was found between the PBD and non-PBD groups $(P=0.19)$. This finding may have been due to the small sample size of the included studies. However, a previous meta-analysis conducted by Moole et al. on 3,532 patients also revealed no reduction in mortality by PBD. ${ }^{19}$

In the present meta-analysis, it was found PBD is associated with significantly lower operative time and estimated blood loss. This finding was probably because the high bilirubin levels in the body can damage the capillary cells and affect the coagulation profile. ${ }^{24}$ This causes difficulty in achieving hemostasis during the operation. Although operation time and intraoperative blood loss are more closely correlated to the surgical expertise and extent of disease, taking measures to reduce the level of serum bilirubin before surgery can reduce operative difficulty to a certain extent.

In the present study, lower incidence of postoperative complications was found in the PBD group. Although the occurrence of complications was correlated to the surgeon's experience, stage of the disease and other patient factors, such as co-morbidities ${ }^{25}$, the reduction in serum bilirubin levels may be beneficial for reducing the incidence of anastomotic leakage ${ }^{26}$. The decrease in bilirubin levels also helps the body overcome the surgical stress. ${ }^{27}$

Although the total length of hospital stay of patients with PBD was slightly higher, when compared to non-PBD patients, the present study revealed that the postoperative hospital stay in the PBD group was significantly shorter than that in the non-PBD group. This observation was probably correlated to the decrease in postoperative complications and faster recovery. Studies have shown that in order to reduce the preoperative hospital stay in patients who received PBD, after a successful PBD, these patients can be encouraged to go home, improve their oral intake, and undergo regular follow-ups of liver functions. Once the serum bilirubin level normalizes, these patients can be readmitted for surgery. ${ }^{28,29}$

There were some limitations in the present study. First, due to the strict selection criteria, many studies with a larger sample size could not be included. Second, the infectious and non-infectious complications could not be separately compared between the PBD and non-PBD groups.

\section{Conclusions}

In conclusion, moderately severe obstructive jaundice patients should receive preoperative biliary drainage. Preoperative biliary drainage can effectively shorten the postoperative hospital stay and operating time, and reduce intraoperative blood loss and complications.

\section{Abbreviations}

preoperative biliary drainage (PBD); pancreatoduodenectomy (PD); odds ratios (OR); confidence intervals (Cl)

\section{Declarations}

Ethics approval and consent to participate: Not applicable.

Consent for publication: Not applicable.

Availability of data and materials: The datasets used and/or analysed during the current study are available from the corresponding author on reasonable request.

Competing interests: The authors declare that they have no competing interests.

Funding: Not applicable. 
Authors' contributions: FW has made substantial contributions to the conception and design of the work. XW and SM have made contributions to the acquisition, analysis, and interpretation of data. YH and KD have made contributions to the creation of new software used in the work. All authors read and approved the final manuscript.

Acknowledgements: Not applicable.

\section{References}

1 Noortje G Rossen,John K Mac Donald,Elisabeth M de Vries,Geert R D’Haens,Willem M de Vos, Erwin G Zoetendal, Cyriel Y Ponsioen. Fecal microbiota transplantation as novel therapy in gastroenterology: A systematic review[J]. World Journal of Gastroenterology. 2015(17).

2 Wadei HM, Mai ML, Ahsan N, Gonwa TA. Hepatorenal syndrome: pathophysiology and management. Clin J Am Soc Nephrol 2006; 1: 1066-1079.

3 Nehez L, Andersson R. Compromise of immune function in obstructive jaundice. Eur J Surg 2002; 168: 315-328.

4 Shaib Y, Rahal MA, Rammal MO, et al. Preoperative biliary drainage for malignant biliary obstruction: results from a national database[J]. J Hepatobiliary Pancreat Sci, 2017, 24(11):637-642.

5 McPherson GA, Benjamin IS, Blumgart LH. Improving renal function in obstructive jaundice without preoperative drainage[J].Lancet, 1984, 323(8375):511512.

6 Moher D, Liberati A, Tetzlaff J, Altman DG; PRISMA Group. Preferred Reporting Items for Systematic Reviews and Meta-Analyses: The PRISMA Statement. PLoS Med 2009; 6: e1000097.

7 Higgins J, Green S (eds). Cochrane Handbook for Systematic Reviews of Interventions Version 5.1.0 (updated March 2011). The Cochrane Collaboration: London, 2011.

8 Sewnath M E, Birjmohun R S, Rauws E A J, et al. The effect of preoperative biliary drainage on postoperative complications after pancreaticoduodenectomy[J]. Journal of the American College of Surgeons, 2001, 192(6): 726-734.

9 Wang X Z,Liu Z C,Zhang X J, et al.Treatment Strategy of Preoperative Bilirubin Abnormality in the Patients with Jaundice Caused by Malignant Obstruction in the Low Bile Duct(Report of 134 Cases)[J].Progress in Modern Biomedicine,2018,18(24):4656-4660+4676.

10 Sang W C, He Q S, Sun C B. Pre-operative biliary drainage in patients with malignant obstructive jaundice of carcinoma of the head of the pancreas[J]. JOURNAL OF SHANDONG UNIVERSITY(HEALTH SCIENCES), 2009, 47(10): 118-121.

11 Chen D, Liang L J, He Y B, et al. Effect of preoperative biliary drainage on the morbidity and mortality of pancreaticoduodenectomy in patients with malignant obstructive jaundice of the lower bile duct [J]. Chin J Gen Surg, 2007, 22(2): 119-122.

12 Wang J X, Kong D. Effect of preoperative biliary drainage and related factors on complications after pancreatoduodenectomy for periampullary carcinoma[J]. Chongqing medicine, 2011, 40(32): 3302-3304.

$13 \mathrm{Bi} \mathrm{X} \mathrm{Y,} \mathrm{Shao} \mathrm{Y} \mathrm{F,} \mathrm{Cai} \mathrm{J} \mathrm{Q,} \mathrm{et} \mathrm{al.} \mathrm{Influence} \mathrm{of} \mathrm{preoperative} \mathrm{biliary} \mathrm{drainage} \mathrm{on} \mathrm{severe} \mathrm{jaundice} \mathrm{patients} \mathrm{undergoing} \mathrm{pancreaticoduodenectomy[J].} \mathrm{Chinese}$ Journal of General Surgery, 2004, 13(6): 441-444.

14 Wen Y Z, Song Y, Peng G F, et al. Effect of preoperative biliary drainage of pancreatoduodenectomy in 65 patients with malignant obstructive jaundice of the lower bile duct[J]. CHINA MEDICINE AND PHARMACY, 2015, 5(10): 142-144.

15 Ma E G. Clinical research of preoperative biliary drainage on malignant obstructive jaundice[D].XinJiang Medical University,2013.『web links: https://kns.cnki.net/KCMS/detail/detail.aspx?

dbcode=CMFD\&dbname=CMFD201401\&filename=1013360595.nh\&v=MDkzMzRxVHJXTTFGckNVUkxPZVp1UnRGeUhoVTd6UFZGMjZIYkMrSHRURnFwRWJC 16 Zhao P. Clinical significance of PBD via PTCD in patients undergoing PD with heavy malignant obstructive jaundice [D].Jilin University,2016.(web links: https://kns.cnki.net/KCMS/detail/detail.aspx?

dbcode=CMFD\&dbname=CMFD201602\&filename=1016083987.nh\&v=MTIwMzZyV00xRnJDVVJMT2VadVJ0RnllaFZMN05WRjI2R0xPd0hkakVxSkViUEISOGV

17 Parildar Z,Cinar C,Barutcuoglu B,et al Effects of percutaneous transhepatic biliary drainage on renal function in patients with obstructive jaundice[J].Diagn Interv Radiol,2010,10(8):4261,1303-3825.

18 TOL J, VAN HOOFT J E, TIMMER R, et al. Metal or plastic stents for preoperative biliary drainage in resectable pancreatic cancer[J]. Gut, 2016, 65(12): 19811987.

19 MOOLE H, BECHTOLD M, PULI S R. Efficacy of preoperative biliary drainage in malignant obstructive jaundice: a metaanalysis and systematic review[J]. World J Surg Oncol, 2016, 14(1): 182.

$20 \mathrm{Li} \mathrm{T}$, Ren K WهHan X W, et al $\mathrm{P}$ Percutaneous transhepatic cholangiobiopsy to determine the pathological cause of anastomotic stenosis after

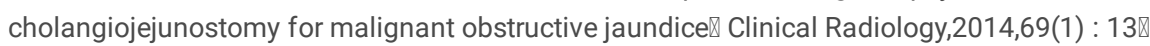

Page 6/17 
21 S tepben PP, Martin SK, Kevin CC, et al.Preoperative biliary drainage : Impact on intraoperative bile cultures and infectious morbidity and mortality after pancreaticoduodenectomy,1999,3(5):496.

22 Calculated Antibiosis of Acute Cholangitis and Cholecystitis[J].Viszeralmediz in,2014,30(5):297-302.

23 Microbial profile and antibiotic sensitivity pattern in bile cultures from endoscopic retrograde cholangiography patients[J]. Muhsin Kaya,Remzi Bestas,Fatma Bacalan,Ferhat Bacaksz,Esma Gülsun Arslan,Mehmet Ali Kaplan[J]. World Journal of Gastroenterology, 2012 , 18 (27) :3585-3589.

24 SCHULZ S, WONG RJ, JANG KY, et al囚 Heme oxygenase-1 deficiency promotes the development of necrotizing enterocolitis-like intestinal injury in a newborn mouse model囚J囚. Am J Physiol Gastrointest Liver Physiol,2013,304(11): 991-1001囚

25 Zang Y J, Yu W L囚Problems needing attention in tumor resection of hilar cholangiocarcinoma and biliary-enteric anastomosis [J].Chinese Journal of Practical Surgery ,2012,32(8):624-626.

26 XU C, HUANG X E, WANG S X, et al. Comparison of infection between internal-external and external percutaneous transhepatic biliary drainage in treating patients with malignant obstructive jaundice[J]. Asian Pac J Cancer Prev, 2015, 16(6): 2543-2546.

27 DUAN F, CUI L, BAI Y, et al. Comparison of efficacy and complications of endoscopic and percutaneous biliary drainage in malignant obstructive jaundice: a systematic review and meta-analysis[J]. Cancer Imaging, 2017, 17(1): 27.

$28 \mathrm{Li} \mathrm{X} \mathrm{Y,Zhao} \mathrm{X,Zheng} \mathrm{P,et} \mathrm{al.Application} \mathrm{of} \mathrm{enhanced} \mathrm{recovery} \mathrm{after} \mathrm{surgery} \mathrm{in} \mathrm{patients} \mathrm{with} \mathrm{duodenopancreatectomy[J].Journal} \mathrm{of} \mathrm{Hepatopancreatobiliary}$ Surgery,2016,28(06):460-464.

29 KEHLET H, WILMORE D W. MULTIMODAL strategies to improve surgical outcome [J]. Am J Surg, 2002, 183(6): 630-641.

\section{Figures}

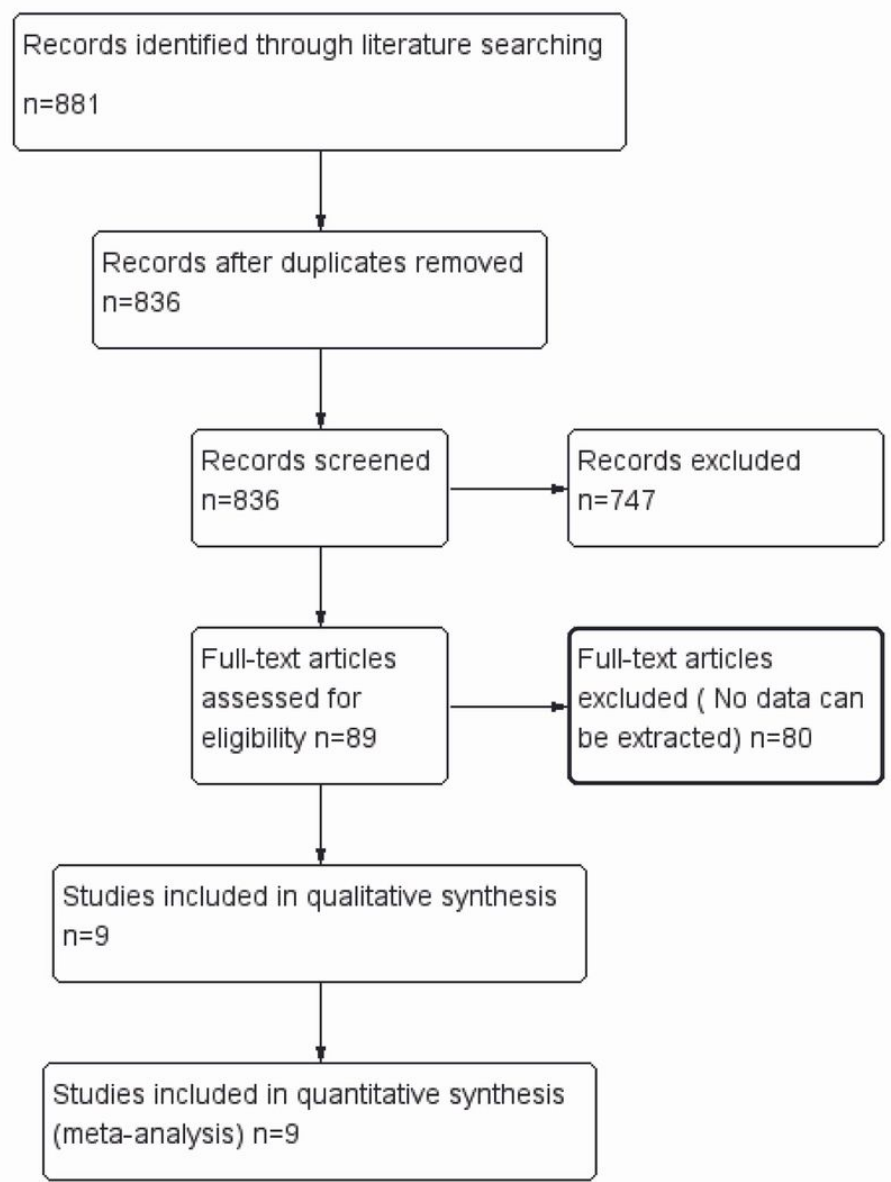

Figure 1 
The flowchart of the selection process for identifying relevant articles for the meta-analysis

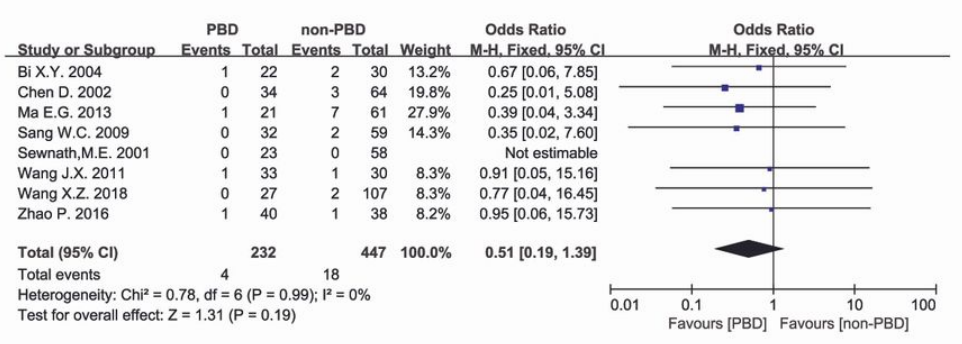

\section{Figure 2}

A forest plot of the individual data and pooled estimate of perioperative mortality in studies that have PBD and non-PBD groups. The Mantel-Haenszel fixed effect model was applied, and the ORs were presented with $95 \% \mathrm{Cl}$. 


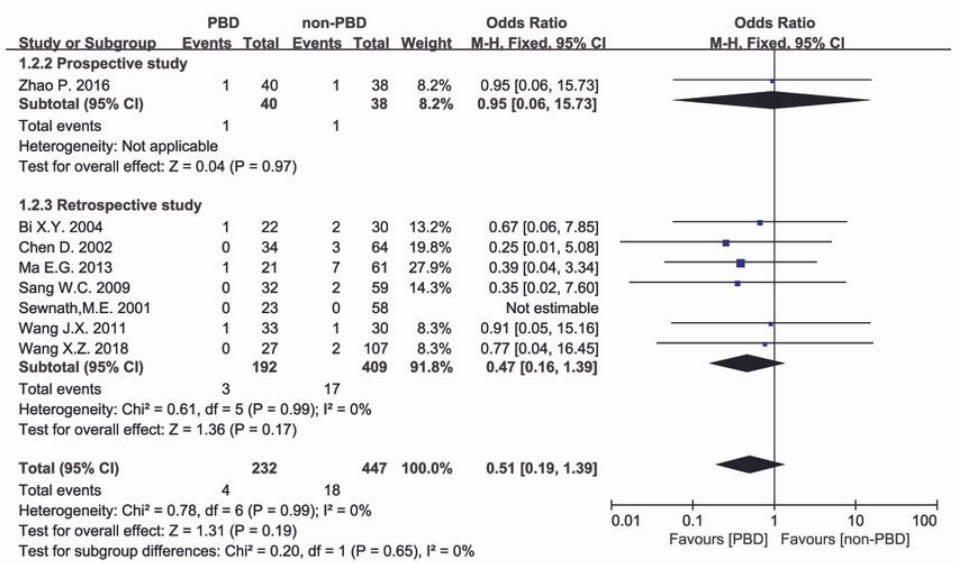

\section{Figure 3}

A forest plot of the pooled estimate of perioperative mortality in prospective and retrospective studies. The Mantel-Haenszel method was applied. 


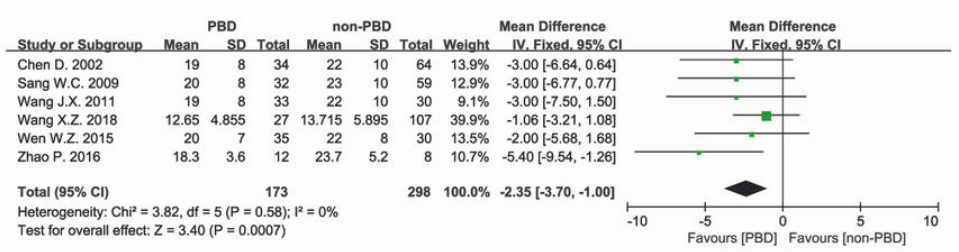

\section{Figure 4}

A forest plot of the individual data and pooled estimate of postoperative hospital stay in the included studies. The inverse variance fixed effect model was applied. The mean difference was presented with $95 \% \mathrm{Cl}$. 


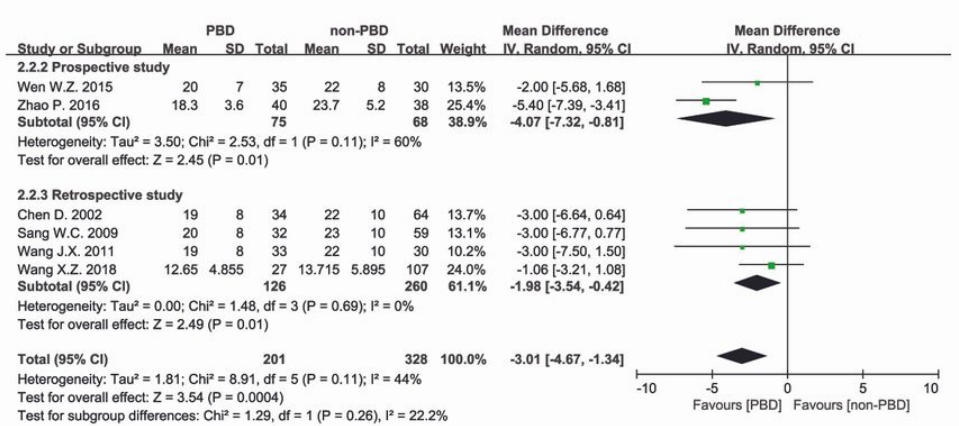

\section{Figure 5}

A forest plot of the pooled estimate of postoperative hospital stay in prospective and retrospective studies. The inverse variance method was applied. 


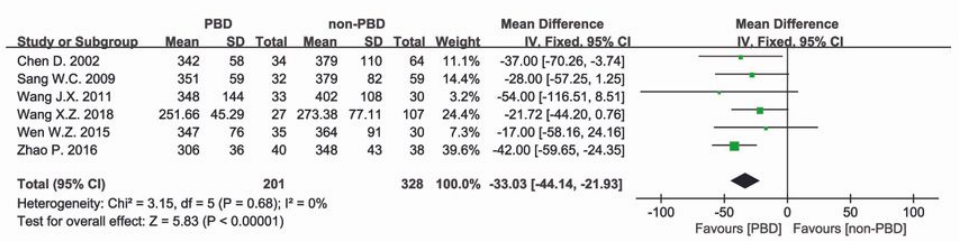

\section{Figure 6}

A forest plot of the individual data and pooled estimate of operative time in the included studies. The inverse variance fixed-effect model was applied. The mean difference was presented with $95 \% \mathrm{Cl}$. 


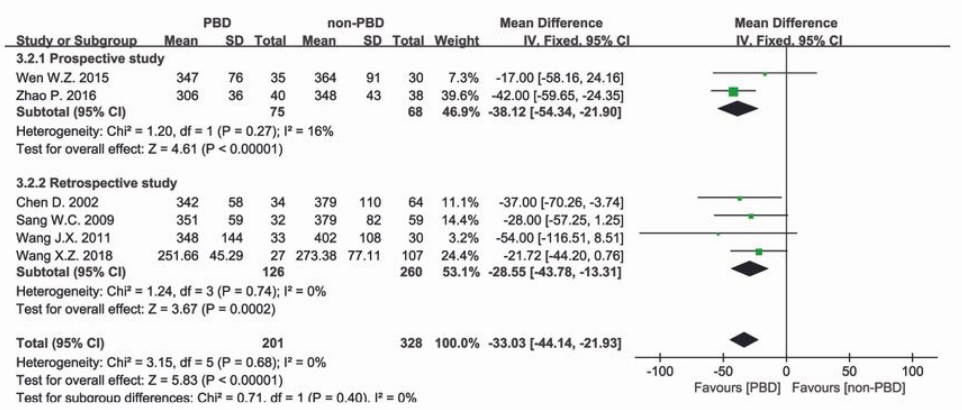

\section{Figure 7}

A forest plot of the pooled estimate of operative time in the prospective and retrospective studies. The inverse variance method was applied. 


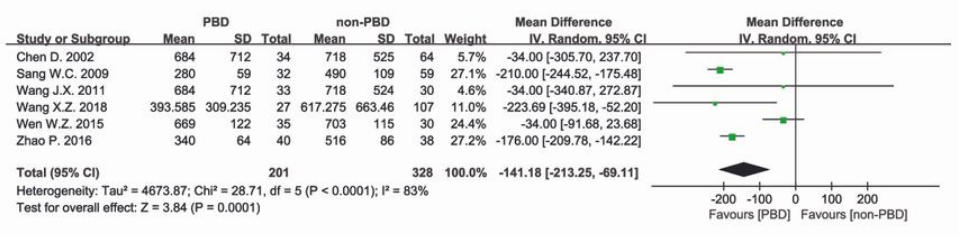

\section{Figure 8}

A forest plot of the individual data and pooled values of estimated blood loss in studies with PBD and non-PBD groups. The inverse variance fixed effects model was applied. The mean difference was presented with $95 \% \mathrm{Cl}$. 


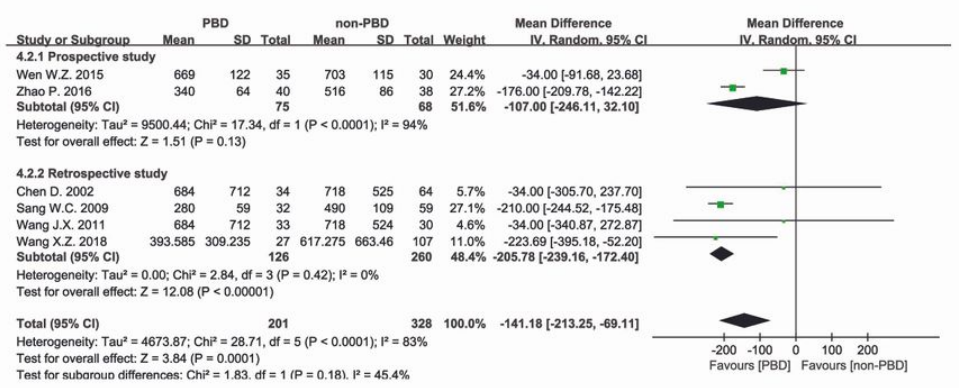

\section{Figure 9}

A forest plot of the pooled values of estimated blood loss in prospective and retrospective studies. The inverse variance method was applied. 


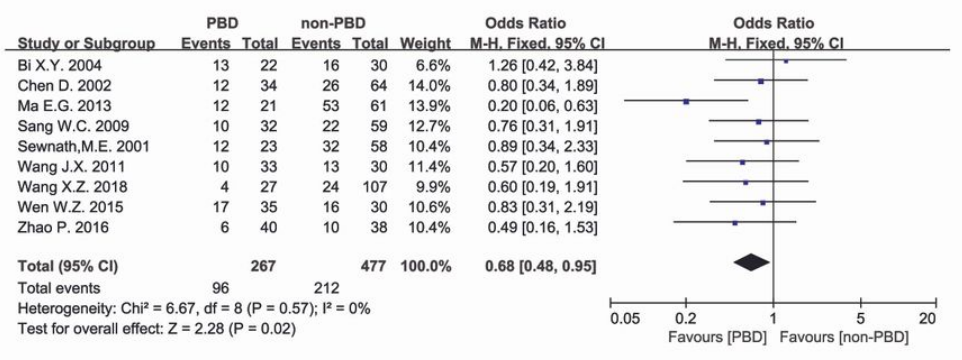

\section{Figure 10}

A forest plot of the individual study proportions and pooled estimate of overall morbidities in the PBD and non-PBD groups. A Mantel-Haenszel fixed effects model was applied. The odds ratios were presented with $95 \% \mathrm{Cl}$. 


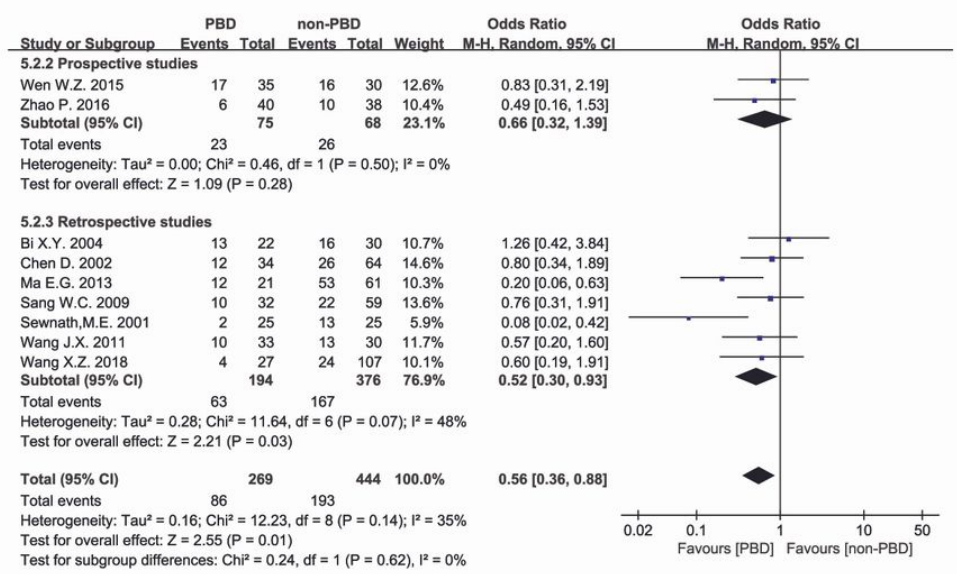

\section{Figure 11}

A forest plot of the pooled values of overall morbidities in prospective and retrospective studies. The Mantel-Haenszel method was applied.

\section{Supplementary Files}

This is a list of supplementary files associated with this preprint. Click to download.

- PRISMAChecklist.docx 